\title{
Generation and Evaluation of Lunar Dust Adhesion Mitigating Materials
}

\author{
Christopher J. Wohl ${ }^{1}$ and John W. Connell ${ }^{2}$ \\ NASA Langley Research Center, MS 226, Hampton, VA, 23681 \\ Yi Lin ${ }^{3}$, Marcus A. Belcher ${ }^{4}$, and Frank L. Palmieri ${ }^{5}$ \\ National Institute of Aerospace, Hampton, VA, 23666 \\ and \\ Brad M. Atkins ${ }^{6}$ \\ Langley Aerospace Research Summer Scholars Program, NASA Langley Research Center, Hampton, VA, 23681
}

\begin{abstract}
Particulate contamination is of concern in a variety of environments. This issue is especially important in confined spaces with highly controlled atmospheres such as space exploration vehicles involved in extraterrestrial surface missions. Lunar dust was a significant challenge for the Apollo astronauts and will be of greater concern for longer duration, future missions. Passive mitigation strategies, those not requiring external energy, may decrease some of these concerns, and have been investigated in this work. A myriad of approaches to modify the surface chemistry and topography of a variety of substrates was investigated. These involved generation of novel materials, photolithographic techniques, and other template approaches. Additionally, single particle and multiple particle methods to quantitatively evaluate the particle-substrate adhesion interactions were developed.
\end{abstract}

\section{Introduction}

D ust in extraterrestrial environments (the Moon, Mars, asteroids, etc.) has been identified by NASA as a major obstacle for the successful completion of future missions. ${ }^{1-6}$ The lunar environment has been the greatest source of evidence to this claim where dust, with diameters $\leq 60 \mu \mathrm{m},{ }^{7}$ comprises approximately $50 \%$ of the lunar regolith. These particulates are porous abrasive, chemically reactive, electrostatically charged, and sometimes magnetic and adhere strongly to exposed surfaces. ${ }^{8,9}$ Lunar dust presented unforeseen challenges during the Apollo missions including abrasion of visors, gloves, and boots, degradation of seals and thermal radiator performance, and respiratory distress. ${ }^{10,11}$ Retro-reflectors deposited on the lunar surface have exhibited a reduction in signal intensity by a factor of ten since their installation in 1969 and $1971 .^{12}$ A plausible explanation for this is lunar dust accumulation on the retro-reflectors' surface. To minimize these issues, novel materials and related technologies need to be developed that passively and actively mitigate lunar dust adhesion. ${ }^{13-16}$ Additionally, techniques to assess the efficacy of mitigation strategies need to be developed. This presentation describes the research efforts at NASA Langley Research Center's Advanced Materials and Processing Branch to address both the generation of materials with intrinsic lunar dust adhesion resistance, and the development of techniques to evaluate the performance of these materials.

\footnotetext{
${ }^{1}$ Materials Research Engineer, Advanced Materials and Processing Branch, Mail Stop 226.

${ }^{2}$ Materials Research Engineer, Advanced Materials and Processing Branch, Mail Stop 226.

${ }^{3}$ Research Scientist, Advanced Materials and Processing Branch, 100 Exploration Way.

${ }^{4}$ Currently employeed at The Boeing Company, Materials and Processes Engineer, Composite Bonding Processes, Everett, WA 98203.

${ }^{5}$ Research Scientist, Advanced Materials and Processing Branch, 100 Exploration Way.

${ }^{6}$ Currently a student at Virginia Polytechnic Institute and State University, Department of Aerospace and Ocean Engineering, 215 Randolf Hall, Blacksburg, VA, 24061.
} 


\section{Low Surface Energy Materials for Adhesion Mitigation}

Mitigation techniques for particulate adhesion can be separated into three different categories: sacrificial coverings and surfaces, active systems, and passive systems. For lunar applications, approaches involving sacrificial surfaces are less practical due to additional weight and time costs. Active mitigation strategies require external energy to remove or prevent particle accumulation and could involve a variety of sources including mechanical power for brush-actuated removal, microwave energy for sintering particulates into larger objects such as roads and other hard surfaces, or electrical power for electrostatic repulsion-driven systems. ${ }^{17}$ Passive mitigation strategies do not require external energy since particle abhesion would be intrinsic to the material. It is conceivable that all three categories of mitigation techniques will be utilized either individually or in a synergistic fashion to overcome the dust problem.

The evaluation of materials for particle adhesion mitigation is not straightforward. There can be several influences that determine the efficacy of a surface for adhesion mitigation including the chemical composition, topography, and mechanical properties of both the surface and the contaminating particulates. Therefore, contact angle goniometry was utilized as an initial approach to evaluate the materials described below. The surface energy of a material, inferred from the contact angle a liquid droplet makes with a surface, was found to show significant correlation with actual particle adhesion. This suggested that, as an initial step, contact angle analysis could be utilized to evaluate the performance of research materials.

\section{A. "Lotus Effect" and POSS Spray Deposition}

Reducing a material's surface energy for particle adhesion mitigation is a strategy used often in nature with the lotus plant, Nelumbo nucifera, receiving perhaps the greatest attention. ${ }^{18,19}$ Here, water droplets form very high contact angles and easily roll off the leaves while simultaneously collecting surface contaminants due to the combination of hierarchical surface topographies and low surface energy chemical coatings. Several researchers have replicated this property, known as the "Lotus Effect". ${ }^{20-22}$ To evaluate the efficacy of this approach for lunar dust adhesion mitigation, new materials were developed and the surfaces of existing materials were modified to correlate surface energy, as determined by contact angle goniometry, and particle adhesion. Initially, surface chemistries and topographies were modified using spray deposition of materials and reactive environmental processes. Polyhedral oligomeric silsesquioxane (POSS) molecules were deposited on polyimide film surfaces and exposure to radio frequency-generated oxygen plasma caused changes in both chemistry and topography (Figure 1A and B). ${ }^{23}$ POSS molecules have been demonstrated to form passivating silica-type coatings on surfaces exposed to atomic oxygen, ${ }^{24-26}$ and this system was selected for investigation as a passive dust mitigation surface with the anticipation that the surface could be modified in a low Earth orbit (LEO) en route to the lunar surface. Formation of a POSS sub-monolayer resulted in reduced surface energy as evidenced by water contact angle goniometry measurements (Figure 1C), suggesting reduced particle adhesion. Exposure to oxygen plasma resulted in additional surface topographic modification, but concomitantly increased the surface energy due to the highly oxidative environment (Figure 1D). Additionally, long-term exposure in the oxidative environment resulted in degradation of both POSS deposits and the polyimide substrate. Initial particle adhesion testing experiments conducted on these surfaces via crude deposition, inversion, and agitation experiments indicated substantial loss of the POSS deposits due to dust particle abrasion. 


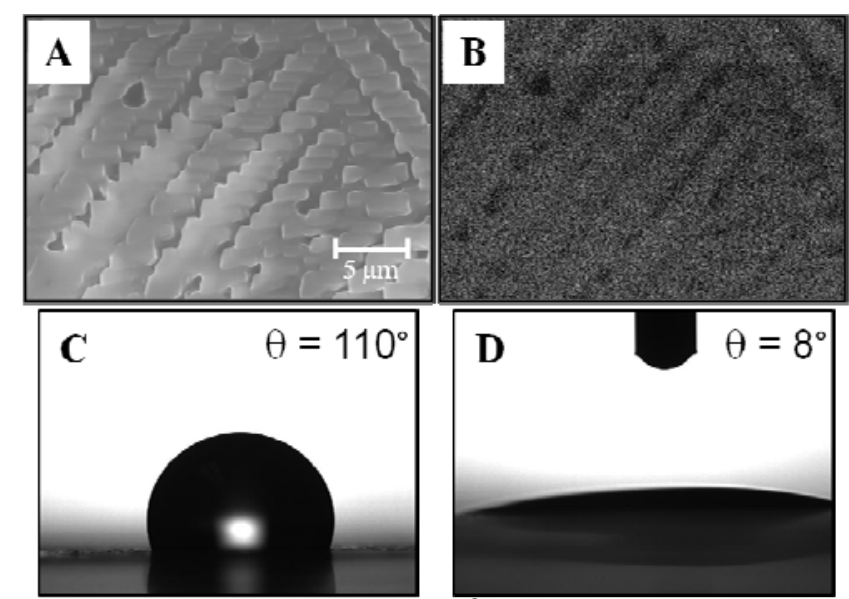

Figure 1. (A) SEM imaging of a POSS-coated Kapton ${ }^{\circledR}$ HN surface where surface topographies resulted from POSS deposition. (B) Identification of POSS deposits was conducted using energy dispersive spectroscopy. In this figure, silicon atoms are indicated by brighter areas with higher concentrations indicated by increased intensity. Water contact angles measured on POSS-coated Kapton HN surfaces before (C) and after (D) oxygen plasma exposure.

\section{B. Copoly(imide)s with Surface Modifying Agents}

More reproducible controlled methods to modify both surface chemistry and topography were subsequently explored. Copolymeric systems were investigated where one of the polymeric components was a surface modifying agent (SMA). SMAs are driven to a polymer's surface by favorable thermodynamic interactions at the polymer-air interface compared to within the polymer matrix (Scheme 1). Thus, surface chemical composition should be enriched with the chemical functionalities present in the SMA component. Copolyimide systems containing butadiene, butadiene acrylonitrile, and siloxane components were investigated (Scheme 2). ${ }^{13}$ These materials were prepared by the reaction of difunctional amine-terminated SMAs with aromatic diamine and dianhydride monomers to generate a polyamide acid intermediate. Films were cast on plate glass using a doctor blade, dried to tack-free in a forced air chamber, and thermally imidized in a nitrogen oven at a maximum temperature of $250^{\circ} \mathrm{C}$. The resultant copolymers contain random imide units with blocks of the SMA component.

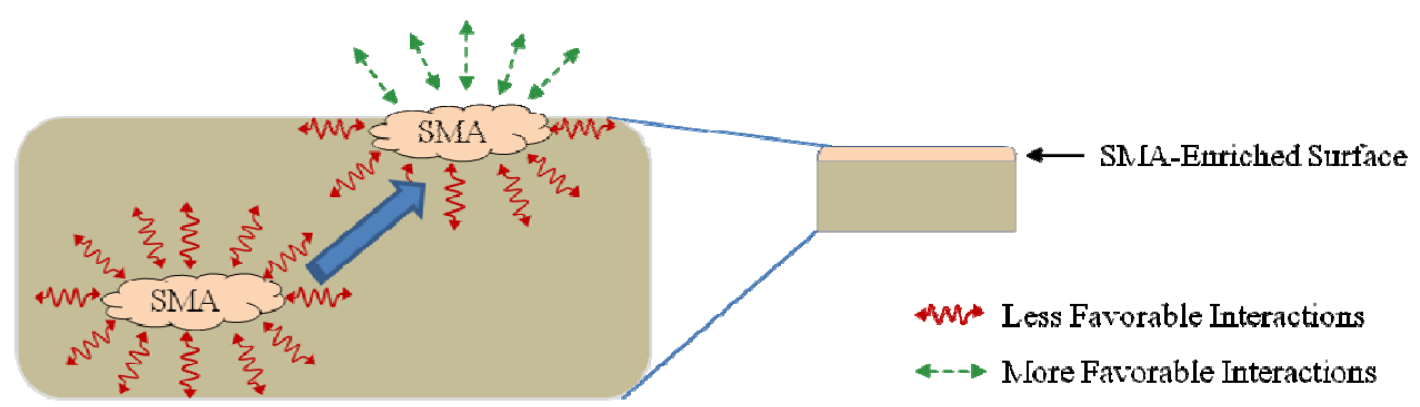

Scheme 1. Surface migration of surface modifying agents (SMA) within a polymer matrix. 
A

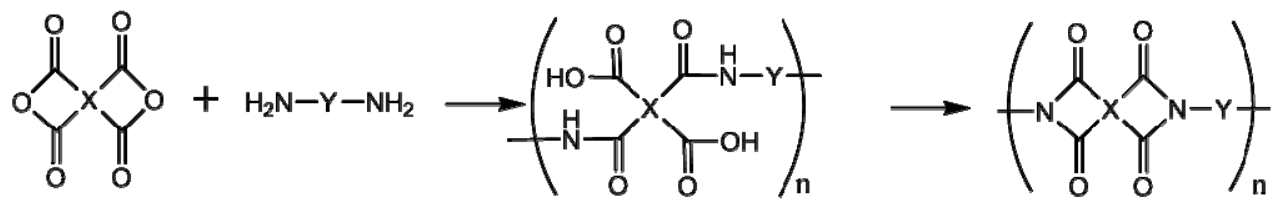<smiles>[2H]NC1CC=C(Oc2ccc(N)cc2)C=C1Oc1cccc(N)c1</smiles><smiles>CC(C)(C)OC(=O)c1ccc(N)cc1</smiles>
Amine-terminated Polybutadiene Acrylonitrile

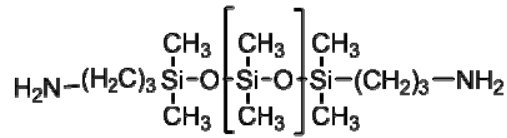
Aminopropyl-terminated Polydimethyl Siloxane

Scheme 2. (A) Polyimide synthesis via condensation polymerization. (B) Aromatic diamine and dianhydride and SMA structures.

Surprisingly, incorporation of both butadiene and butadiene acrylonitrile moieties resulted in an increase in surface energy with water contact angles averaging $82^{\circ}$, compared to $87^{\circ}$ for the homopolyimides (Figure 2 ). ${ }^{27}$ Incorporation of siloxane functionalities, however, resulted in a significant decrease in surface energy. For example, copoly(imide siloxane)s exhibited, on average, advancing water contact angles higher than the homopolyimide material, $110^{\circ}$ and $87^{\circ}$, respectively (Figure 2). Varying the siloxane segment length resulted in changes to film morphology, thermal, mechanical, and surface properties. ${ }^{28}$ As can be seen in Figure 3, increased siloxane oligomer length resulted in increased water contact angle. This suggested the presence of greater surface concentration or/and increased surface thickness of the siloxane enriched region resulting in surface energy decrease. The tensile modulus of the copoly(imide siloxane)s was measured to be less than that of the homopolyimide with the difference increasing with siloxane olgimer length. Interestingly, the tensile modulus increased for the copoly(imide siloxane) with the greatest siloxane oligomer length, relative to the copoly(imide siloxane) prepared with the second largest siloxane oligomer length, suggesting a change in the film morphology. Siloxane surface migration was verified using X-ray photoelectron spectroscopy (XPS, data not shown). 


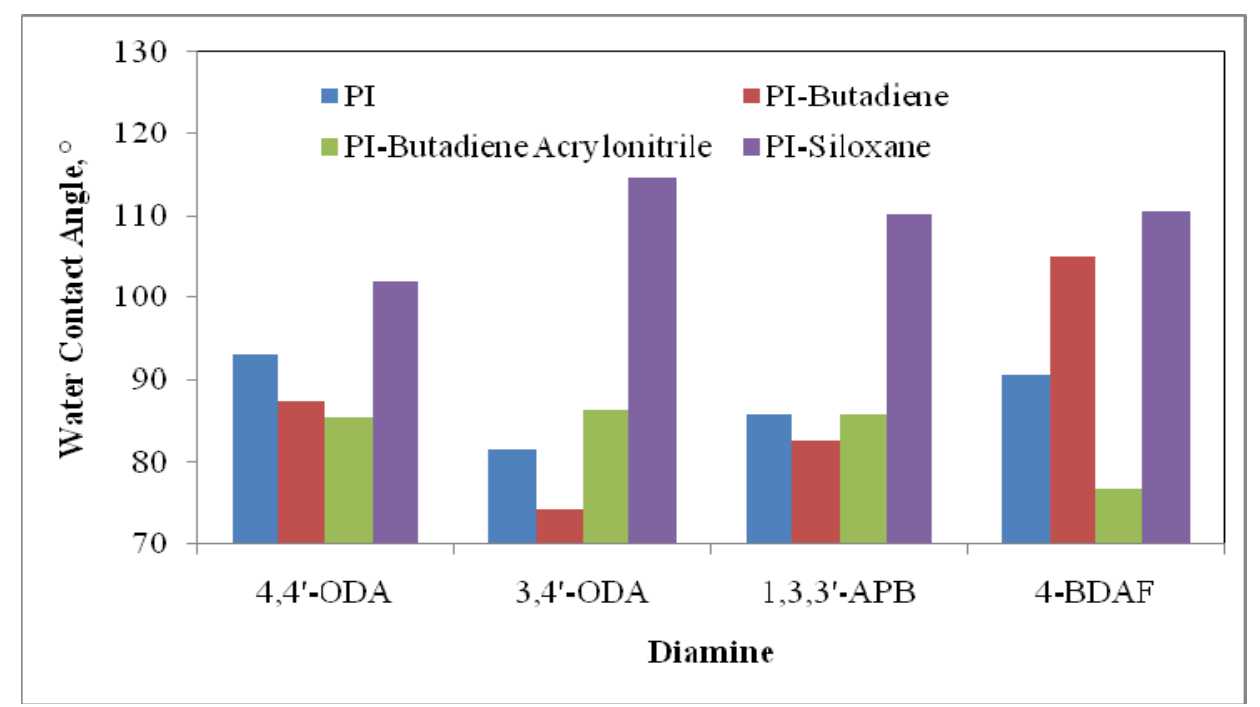

Figure 2. Water contact angle values measured on the "air" side of polyimide (PI) and copolyimide films.

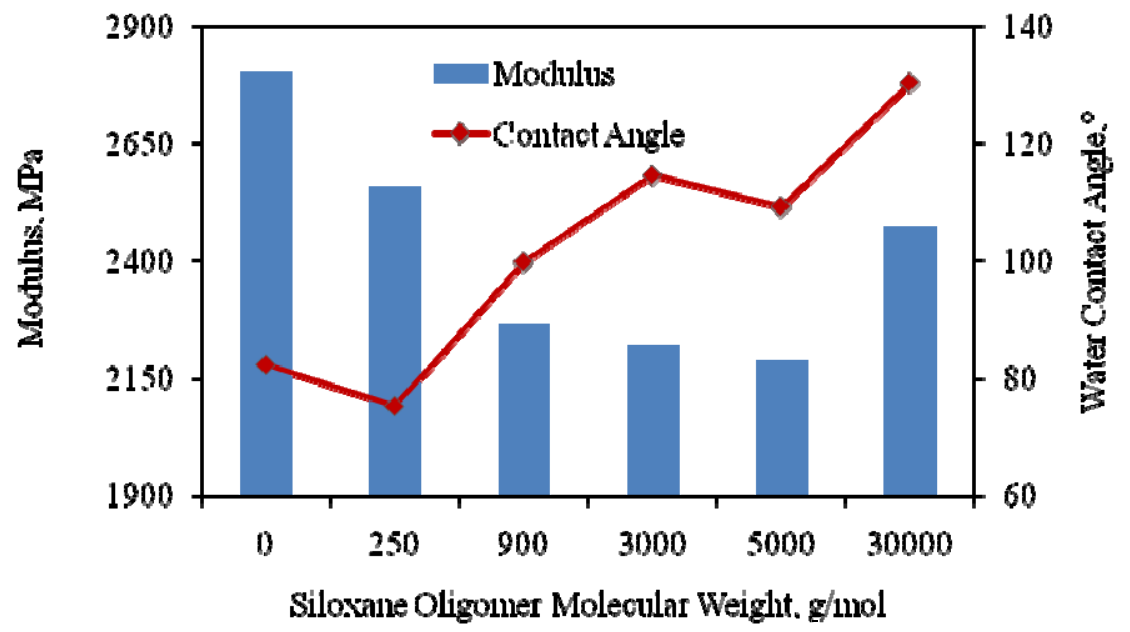

Figure 3. The modulus (left axis) and contact angle values (right axis) of copoly(imide siloxane) films.

\section{Reducing Available Surface Area using Photolithography}

A reduction in available surface area may result in Cassie-Baxter wetting states, where air is trapped beneath water droplets resting on the surface. Controlled alteration of surface topography may also contribute to particulate adhesion mitigation and several techniques were investigated to explore this possibility: photolithography, laser ablation patterning, and templated melt-extrusion. Photo-masks consisting of square arrays were made with varying percentages of surface area coverage $(11-44 \%)$. Several different polymeric materials were processed using film deposition, positive photoresist, and reactive ion etching forming a square-pillar array surface topography (Figure 4A). These surfaces exhibited increased water contact angles, but did not demonstrate repeatable performance due to instability of the pillar topographies. A similar approach was applied to silicon surfaces followed by subsequently coating with a low surface energy moeity, (tridecafluoro-1,1,2,2-tetrahydrooctyl)-trichlorosilane. These surfaces demonstrated a high surface energy once the topographies were generated (i.e., water contact angles $<10^{\circ}$ ) and dramatically lower surface energies after chemical treatment $\left(113^{\circ}-135^{\circ}\right)$ (Figure 4B). 

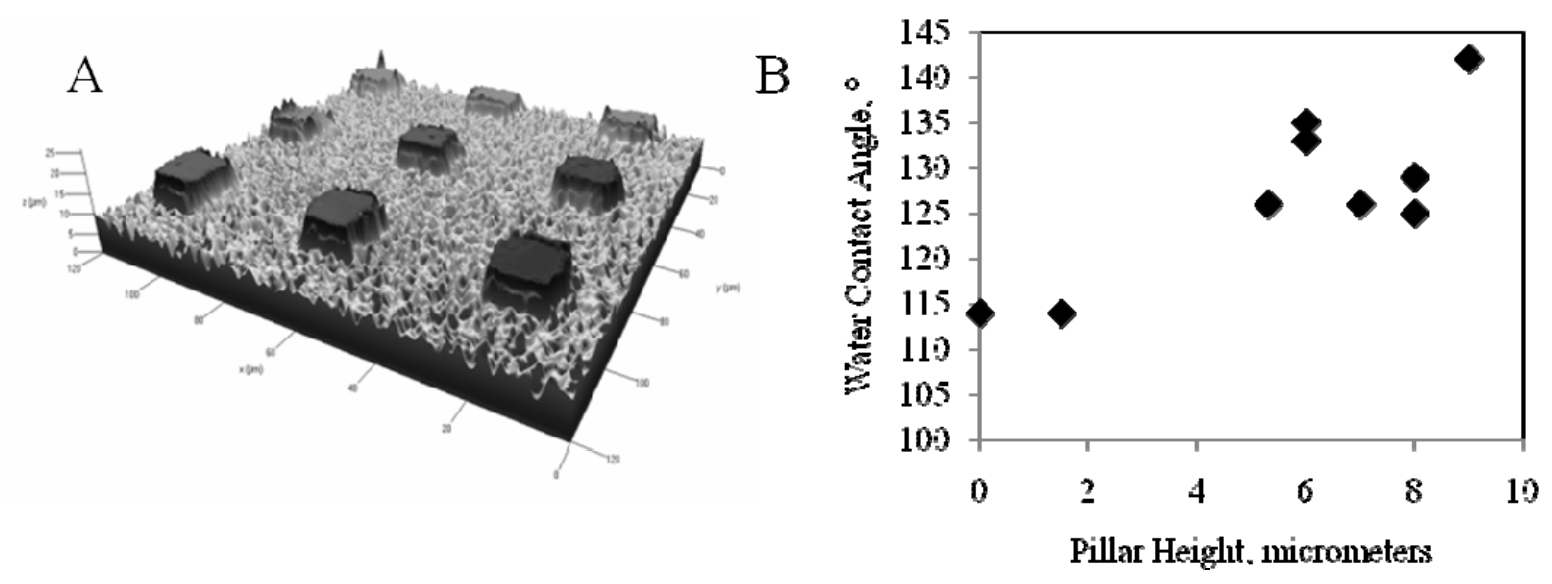

Figure 4. (A) A confocal micrograph of a copolymer film patterned using photolithography. The pillar heights are approximately 10 micrometers. (B) Water contact angle values for silicon wafers with square pillar arrays generated by photolithography and silane treatment.

\section{Reducing Available Surface Area using Laser Ablation Patterning}

Laser ablation patterning has been demonstrated in this laboratory to be a very useful technique for accurately altering the surface topography of a variety of materials, including metals, polymers, ceramics, and composites. ${ }^{13,29,30}$ Laser ablative patterning was achieved using an Avia frequency-tripled Nd:YAG laser (7W, $\lambda=$ $355 \mathrm{~nm}$ ). After evaluating a series of surface patterns, a cross-hatch pattern was found to be the most effective for generation of low surface energy materials. The surface topography was observed using confocal microscopy, the interstices between the lines of the cross-hatch pattern appeared as conical pillars on laser ablation patterned surfaces (Figure 5A). Typical feature heights were 15 microns and were achieved with pulse energies of approximately $25 \mu \mathrm{J}$ per pulse and a beam diameter of $25 \mu \mathrm{m}$. The changes in surface topography resulted in dramatic changes in the surface energy of the materials as indicated by contact angle goniometry with water contact angle values approaching $175^{\circ}$ for patterned surfaces. Water droplets would not spontaneously detach from the contact angle goniometer delivery syringe onto laser ablation patterned copoly(imide siloxane) surfaces (Figure 5B). Therefore, a narrow glass fiber was used as an aid in detaching the droplet.
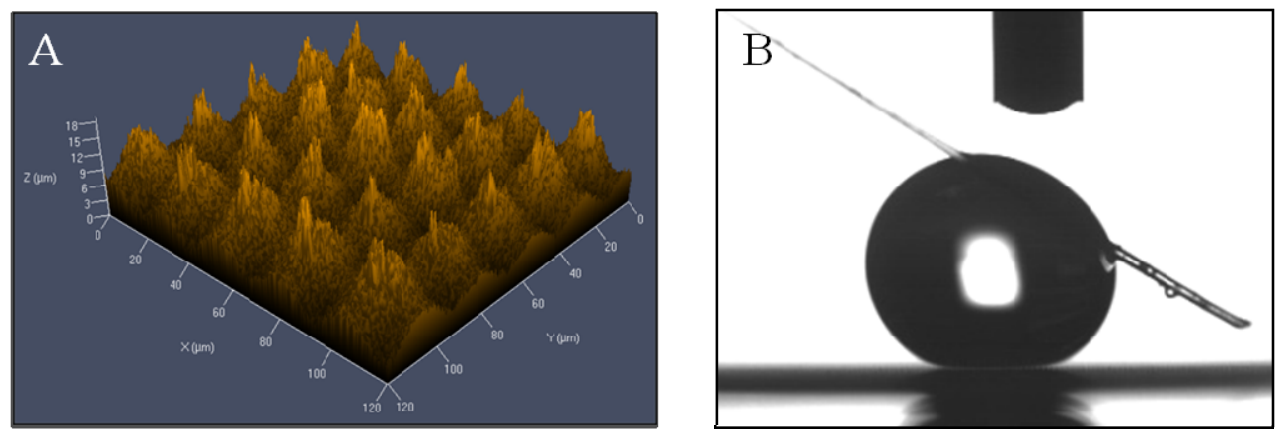

Figure 5. (A) A confocal micrograph of a copoly(imide siloxane) surface after laser ablation patterning. (B) Light can be seen underneath the water droplet on a laser patterned copoly(imide siloxane) surface even though there was sufficient force from the glass fiber to distort the drop shape.

\section{E. Gecko-toe Biomimetics}

The incredible climbing ability of geckos on vertical surfaces, even in dusty environments, has been linked to the hierarchical surface topographies found on their toes. Generation of biomimetic structures that emulate these properties is particularly attractive for lunar dust mitigation since contact self-cleaning can be achieved in dry environments. To reproduce gecko toe properties, polypropylene films were modified using commercially available 
polycarbonate filter membranes as sacrificial templates. Surface topographies were varied from 0.3-10 $\mu \mathrm{m}$ with single and dual length scale surface topographies being generated (Figure 6A). Limitation of the available surface area as a result of these topographies increased the water contact angle values from $107^{\circ}$ to $164^{\circ}$ (Figure 6B). Geckos remove particles from their toes simply by stepping on clean surfaces and a similar methodology was investigated for the gecko mimetic polypropylene surfaces contaminated with Zeeospheres ${ }^{\mathrm{TM}}$ (silica-alumina microspheres, $\sim 1-5 \mu \mathrm{m}$ ). The surfaces, coated with Zeeospheres ${ }^{\mathrm{TM}}$, were translated across a clean surface in 5 "steps" consisting of contact with the surface, downward force with slight shear in a single direction, and removal from the surface in a directional peeling fashion. Although the surfaces appeared to be particle free after the 5 step cleaning procedure, high-resolution scanning electron microscopic (HRSEM) imaging revealed particles persisting on the surface and entangled in the hierarchical structures. A representative statistical analysis of the remaining particles on a gecko-toe biomimetic surface is shown as Figure 6C. The mean particle size of particles remaining on the surface depended on the size of the surface topographies and the mechanism of adhesion. Surfaces with numerous small pillars exhibited entanglement of larger particles due to the low modulus of polypropylene, while smaller particles remained on surfaces with larger pillar features as a result of deposition between pillars. Although the mean particle size was reduced from that for the original Zeeospheres ${ }^{\mathrm{TM}}$ size distribution, the degree of surface contamination after the 5 step cleaning procedure indicated the necessity to either increase the modulus of the surface material or incorporate mixed size topographical features to mitigate the various adhesion mechanisms. Both of these efforts are currently being pursued.
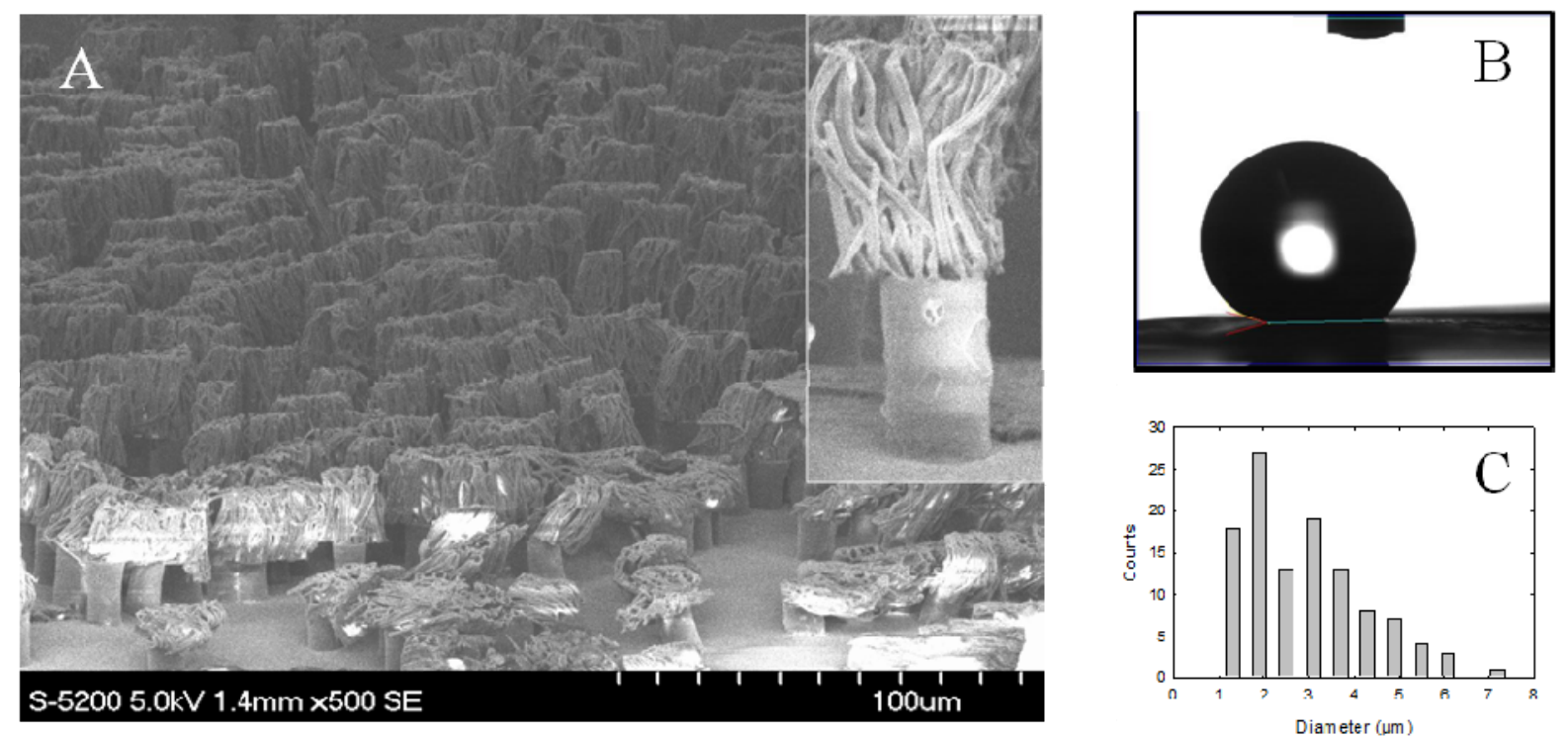

Figure 6. (A) HRSEM image of a hierarchical gecko toe biomimetic polypropylene surface. Inset is a higher magnification of a single hierarchical pillar. (B) Water contact angles increased significantly on these surfaces. (C) Representative remaining particle statistics on a hierarchical gecko-toe biomimetic surface after undergoing 5 cleaning "steps."

\section{Quantitative Adhesion Force Measurement Methods}

Two approaches were utilized to ascertain the effectiveness of surface modified materials to mitigate particulate adhesion focusing on either single particle interactions or multiple particle interactions. Single particle adhesion force measurements were made using atomic force microscopy (AFM) $;{ }^{31}$ multiple particle adhesion utilized a custom-built particle adhesion testing device.

\section{A. Adhesion Force Microscopy}

To determine adhesion force values using AFM, silicon nitride cantilevers, with a radius of curvature of $\sim 10 \mathrm{~nm}$, were translated towards the interrogated surface until van der Waals forces caused the cantilever to "jump" into contact (Figure 7A, I). The cantilever was then brought closer until repulsive forces caused deflection of the cantilever (Figure 7A, II), at which point the motion of the cantilever was reversed. The deflection of the cantilever was recorded as the separation distance increased until the cantilever detached from the sample surface (Figure 7A, III). The deflection force of the cantilever, which can be related to the cantilever's spring constant, was used to 
calculate the work of adhesion. Copolyimide materials with lower modulus exhibited increased adhesion force values, relative to Kapton ${ }^{\circledR} \mathrm{HN}$, likely from an increase in the AFM cantilever embedding depth into a softer material (Figure 7B). Differences in bulk and surface elasticity can contribute to significantly different properties and a recent publication described the use of reduced modulus values to account for this behavior. ${ }^{32}$ Surfaces topographically modified using laser ablation patterning exhibited a reduction in adhesion force (to approximately $25 \%$ of that on the unpatterned surface).
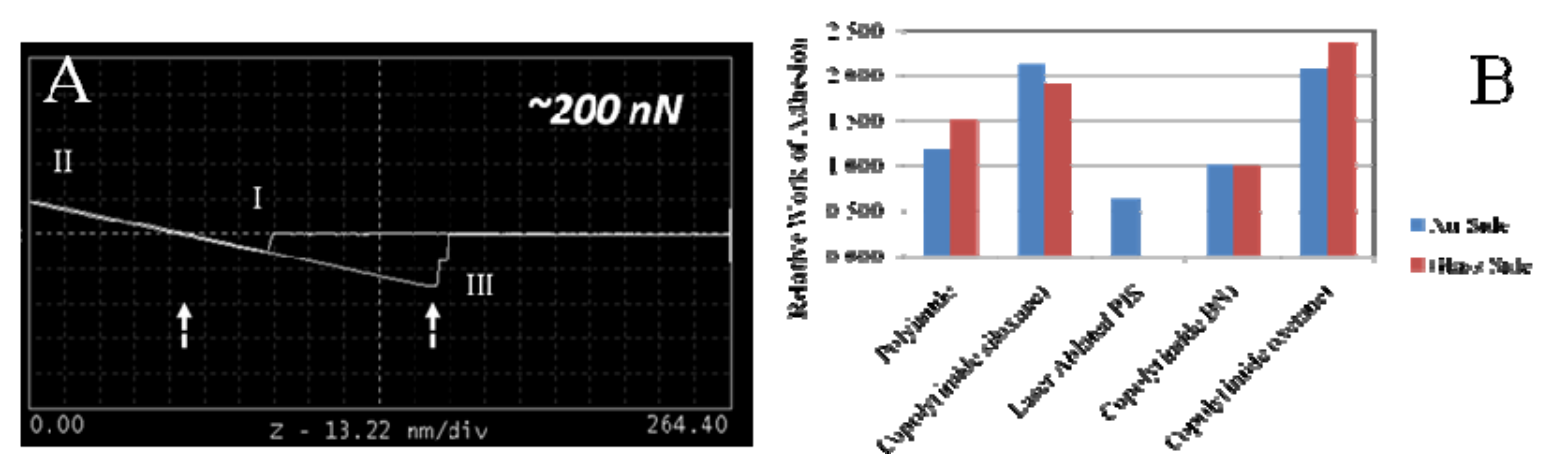

Figure 7. (A) A sample deflection/displacement curve collected during an AFM measurement. The distance between the two white arrows (i.e., the cantilever displacement) was used to calculate adhesion force. (B) Work of adhesion values, relative to Kapton ${ }^{\circledR} \mathrm{HN}$ determined on the air and glass side of copolyimide films.

\section{B. Multiple Particle Adhesion Measurements}

Multiple particle adhesion was evaluated using a custom-built particle adhesion testing device ${ }^{33}$ that utilized sonication to detach particles adhered to a sample surface (Figure 8). ${ }^{34}$ The detached particles were gravitationally fed into an optical particle counter (OPC) that detected the size and number of detached particles. From the OPC data, adhesion force values were determined for an array of substrates and particle compositions using Johnson Kendall Roberts (JKR) theory, which is an extension of Hertz theory taking surface energy and elastic deformation interactions within the contact area into account. ${ }^{35}$ 

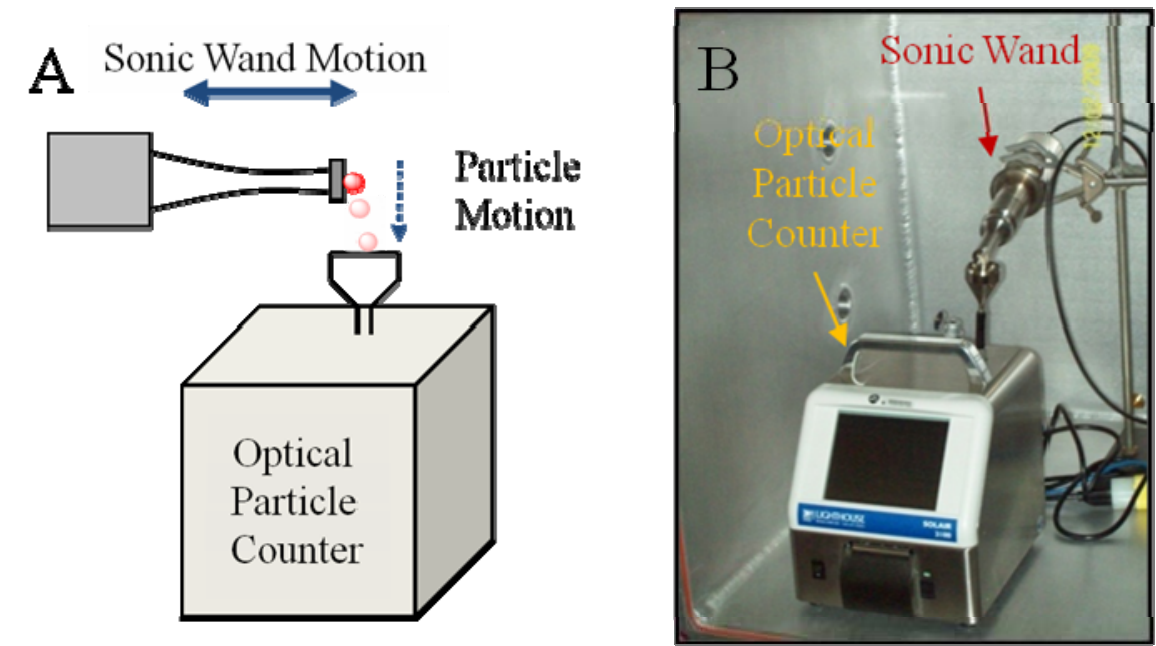

Figure 8. A) A schematic of the particle adhesion testing device. B) The components of the particle adhesion testing device included a $0.23 \mathrm{~m}^{3}$ aluminum environmental chamber, a sonic wand (red), and an optical particle counter (orange). The device was constructed to enable several environmental conditions to be altered (i.e., electric fields, humidity and pressure levels, atmospheric composition, UV exposure).

According to JKR theory, the adhesion force can be related to the work of adhesion by:

$$
F_{a d h}=\frac{3}{2} \pi R W_{a}
$$

The work of adhesion can then be used to calculate the contact radius, $a_{o}$, of particles adhered to the surface according to:

$$
a_{0}=\left(\frac{6 \pi R^{2} W_{a}}{K}\right)^{1 / 3}
$$

where $K$ is:

$$
K=\left(\frac{4}{3}\right)\left(\frac{1-v_{1}^{2}}{E_{1}}+\frac{1-v_{2}^{2}}{E_{2}}\right)^{-1}
$$

Thus, the contact radius and ultimately the work of adhesion depend upon the Poisson's ratio, $v_{i}$, and the elastic modulus, $E_{i}$, of both the substrate and the particle. Using this analysis, a series of metallic and polymeric surfaces were tested using the adhesion device to determine $W_{a}$ values with soda-lime glass spheres of various diameters (4$70 \mu \mathrm{m})$. The experimentally determined $W_{a}$ values were next compared to calculated $W_{a}$ values derived from contact angle measurements. The work of adhesion between two dissimilar surfaces can be thermodynamically defined as:

$$
W_{a}=\gamma_{1}+\gamma_{2}-\gamma_{12}
$$

where $\gamma_{1}$ and $\gamma_{2}$ are the surface energies of the two distinct surfaces, particle and substrate respectively, and $\gamma_{12}$ is the interfacial interaction energy. ${ }^{36}$ Since the interfacial interaction energy term cannot be determined using contact 
angle measurements, one approach to calculate the work of adhesion between surfaces is to partition the surface energy of a material into dispersive and polar contributions, $\gamma^{d}$ and $\gamma^{p}$ respectively, where the total surface energy, $\gamma^{\text {tot }}$, is simply the sum. Then, the work of adhesion between these surfaces is approximated as the sum of the geometric means of these components. ${ }^{37}$

$$
W_{a}=2\left(\gamma_{1}^{d} \gamma_{2}^{d}\right)^{1 / 2}+2\left(\gamma_{1}^{p} \gamma_{2}^{p}\right)^{1 / 2}
$$

To calculate $W_{a}$ using surface energy contributions, contact angle goniometry experiments were performed on each of the investigated surfaces with a microscope slide as a representative of the soda-lime glass particles (data not shown). Contact angle values were determined on each surface using water, ethylene glycol, and methylene iodide which were used to calculate total, dispersive, and polar surface energies using the Kaelble plot according to Eq. $6 .{ }^{37}$

$$
(1+\cos \theta) \frac{\gamma_{L}}{2\left(\gamma_{L}^{d}\right)^{1 / 2}}=\left(\gamma_{S}^{d}\right)^{1 / 2}+\left(\gamma_{S}^{p}\right)^{1 / 2}\left(\frac{\gamma_{L}^{p}}{\gamma_{L}^{d}}\right)^{1 / 2}
$$

The most significant result from these tests was a correlation between surface energy values, determined by contact angle goniometry, and adhesion force values (Figure 9). The identity of the slope and intercept of this linear relationship is still being investigated.

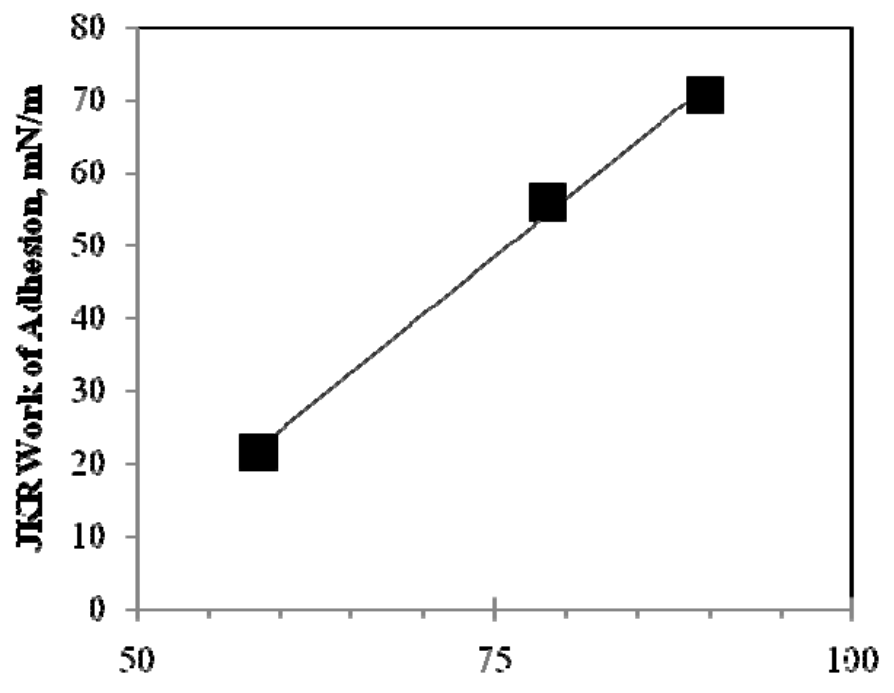

Thermodynamic Work of Adhesion, mNim

Figure 9. Comparison of work of adhesion, $W_{a}$, values determined experimentally ( $y$-axis) and calculated using contact angle goniometry ( $x$-axis).

Using the custom-built adhesion testing device, surface topographical modification was again found to reduce adhesion forces. Silastic ${ }^{\circledR}$ T2, a two-part silicone, was adhered to the sonication device as either a pristine (flat) surface or a patterned surface, which was the negative of a laser ablation patterned aluminum coupon. These surfaces were then coated with soda-lime glass particles and subjected to agitation via sonication. As can be seen in Table 1, the patterned surfaces exhibited dramatically lower adhesion force values, greater surface clearance, and lower embedding depths compared to the flat surface. Similar relationships have been observed for laser ablation patterned materials (data not shown). 
Table 1. Force of adhesion data determined for unpatterned and patterned Silastic ${ }^{\circledR}$ T2 surfaces coated with soda-lime glass microparticles.

\begin{tabular}{|c|c|c|c|c|}
\hline Surface & $\begin{array}{c}\text { Particle } \\
\text { Radius }(\mu \mathrm{m})\end{array}$ & $\begin{array}{c}\text { Adhesion } \\
\text { Force }(\mu \mathrm{N})\end{array}$ & Clear\% ${ }^{\mathrm{a}}$ & $\begin{array}{c}\text { Contact Radius } \\
(\mu \mathrm{m})^{\mathrm{b}}\end{array}$ \\
\hline \multirow{2}{*}{ Silastic $^{\circledR} \mathrm{T} 2$} & 12.5 & 40 & $<50$ & 8.4 \\
\hline & 20 & 150 & $<50$ & 15.3 \\
\hline \multirow{2}{*}{$\begin{array}{c}\text { Silastic }^{\circledR} \mathrm{T} 2, \\
\text { patterned }\end{array}$} & 12.5 & 6.4 & 75 & 4.6 \\
\hline & 20 & 86 & 100 & 12.7 \\
\hline
\end{tabular}

\section{Conclusion}

Lunar surface missions will require an array of different technologies and materials to adequately address the challenges presented by lunar dust. The results discussed here yield several conclusions regarding successful materials for lunar dust adhesion mitigation. First, the material must not possess a highly pliable surface, e.g., a silicone-type material, that would allow significant embedding of incident particulates. Second, surface chemical functionalities must be non-polar or result in a low surface energy material, e.g. fluorinated functionalities. Surface topographies, of proper dimensions, are likely to minimize adhesion forces. Finally, contact angle goniometry was demonstrated to be a useful tool to evaluate the efficacy of materials for prevention of particulate adhesion in both terrestrial and lunar applications. Efforts to determine the impact of electrostatic charging and to increase abrasion resistance are currently underway.

\section{Acknowledgments}

The authors would like to acknowledge several members of the NASA Langley Advanced Materials and Processing Branch including Drs. Jeffrey Hinkley, Joseph Smith Jr., and Sayata Ghose. The authors would also like to acknowledge several members of the NASA Langley Fabrication Technology Development Branch including Nancy Holloway, John Hopkins, and Vincent Cruz. The authors would like to acknowledge Carlos Calle and other researchers in the Electrostatics and Surface Physics Laboratory at NASA Kennedy Space Center. The authors would also like to acknowledge funding from the NASA Langley Creative and Innovative Program as well as the Langley Aerospace Research Summer Scholars program for support of Brad Atkins.

\section{References}

(1) Stubbs, T., Vondrak, R. and Farrell, W., "Impact of Dust on Lunar Exploration," Dust in Planetary Systems, Kauai, Hawaii, USA, 2005.

(2) Kobrick, R. L. and Klaus, D. M., "Characterizing Physical Properties and Induced Motion of Lunar Dust Affecting Surface Exploration Missions.," 39th Lunar and Planetary Science Conference, League City, TX, 2008.

(3) Gaier, J., "The Effects of Lunar Dust on Eva Systems During the Apollo Missions," National Aeronautics and Space Administration, NASA/TM-213610, 2005.

(4) Gaier, J. R., Siamidis, J. and Larkin, E. M. G., "Effect of Simulated Lunar Dust on the Properties of Thermal Control Surfaces," J Spacecraft Rockets, 47,2010, pp. 147 - 152.

(5) Khan-Mayberry, N., "The Lunar Environment: Determining the Health Effects of Exposure to Moon Dusts," Acta Astronautica, 63,2008, pp. 1006-1014.

(6) Holland, J. M. and Simmonds, R. C., "The Mammalian Response to Lunar Particulates," Space Life Sciences, 4,1973, pp. $97-109$.

(7) Taylor, L. A., Schmitt, H. H., Carrier, W. D. and Nakagawa, M., "The Lunar Dust Problem: From Liability to Asset," 1st Space Exploration Conference: Continuing the Voyage of Discovery, Orlando, Florida, 2005.

(8) Lee, L.-H., "Adhesion and Cohesion Mechanism of Lunar Dust on the Moon's Surface," Journal of Adhesion Science and Technology, 9,1995, pp. 1103 - 1124.

(9) Walton, O. R., "Adhesion of Lunar Dust," National Aeronautics and Space Administration, NASA/CR-214685, 2007.

(10) Kobrick, R. L., Budinski, K. G., Street Jr, K. W. and Klaus, D. M., "Three-Body Abrasion Testing Using Lunar Dust Simulants to Evaluate Surface System Materials," National Aeronautics and Space Administration, NASA/TM-216781, 2010.

(11) Liu, Y., Schnare, D. W., Eimer, B. C. and Taylor, L. A., "Dry Separation of Respirable Lunar Dust: Providing Samples for the Lunar Airborne Dust Toxicity Advisory Group," Planet. Space Sci., 56,2008, pp. 1517-1523.

11

American Institute of Aeronautics and Astronautics 
(12) Murphy Jr., T. W., Adelberger, E. G., Battat, J. B. R., Hoyle, C. D., McMillan, R. J., Michelsen, E. L., Samad, R. L., Stubbs, C. W. and Swanson, H. E., "Long-Term Degradation of Optical Devices on the Moon.," Icarus, 208,2010, pp. 3135 .

(13) Wohl, C. J., Belcher, M. A., Chen, L. and Connell, J. W., "Laser Ablative Patterning of Copoly(Imide Siloxane)S Generating Superhydrophobic Surfaces," Langmuir, 26,2010, pp. 11469-11478.

(14) Gaier, J. R. and Jaworske, D. A., "Lunar Dust on Heat Rejection System Surfaces: Problems and Prospects.," National Aeronautics and Space Administration, NASA/TM-214814, 2007.

(15) Wilson, T. L. and Wilson, K. B., "Regolith Sintering: A Solution to Lunar Dust Mitigation," 36th Lunar and Planetary Science Conference, League City, Texas, 2005.

(16) Eimer, B. C. and Taylor, L. A., "Dust Mitigation: Lunar Air Filtration with a Permanent-Magnet System (Laf-Pms)," 38th Lunar and Planetary Science Conference, League City, Texas, 2007.

(17) Immer, C., Starnes, J., Michalenko, M., Calle, C. and Mazumder, M., "Electrostatic Screen for Transport of Martian and Lunar Regolith," 37th Lunar and Planetary Science Conference, League City, Texas, 2006.

(18) Barthlott, W. and Neinhuis, C., "Purity of the Sacred Lotus, or Escape from Contamination in Biological Surfaces.," Planta, 202,1997, pp. 1 - 8.

(19) Neinhuis, C. and Barthlott, W., "Characterization and Distribution of Water-Repellent, Self-Cleaning Plant Surfaces," Annals of Botany, 79,1997, pp. 667 - 677.

(20) Liu, M., Zheng, Y., Zhai, J. and Jiang, L., "Bioinspired Super-Antiwetting Interfaces with Special Liquid-Solid Adhesion," Accounts of Chemical Research, 43,2010, pp. 368-377.

(21) Ma, M. and Hill, R., "Superhydrophobic Surfaces," Current Opinions in Colloid and Interface Science, 11,2006, pp. 193202.

(22) Roach, P., Shirtcliffe, N. and Newton, M., "Progress in Superhydrophobic Surface Development," Soft Matter, 4,2008, pp. 224-240.

(23) Wohl, C. J., Belcher, M. A., Ghose, S. and Connell, J. W., "Modification of the Surface Properties of Polyimide Films Using Poss Deposition and Oxygen Plasma Exposure " Appl. Surf. Sci., 255,2009, pp. 8135 - 8144.

(24) Augustine, B., Hughes, W. C., Zimmermann, K., Figueiredo, A., Guo, X., Chusuei, C. and Maidment, J., "Plasma Surface Modification and Characterization of Poss-Based Nanocomposite Polymeric Thin Films," Langmuir, 23,2007, pp. 4346 4350.

(25) Brunsvold, A. L., Minton, T., Gouzman, I., Grossman, E. and Gonzalez, R. I., "An Investigation of the Resistance of Polyhedral Oligomeric Silsesquioxane Polyimide to Atomic-Oxygen Attack.," High Performance Polymers, 16,2004, pp. $303-318$.

(26) Phillips, S., Haddad, T. and Tomczak, S., "Developments in Nanoscience: Polyhedral Oligomeric Silsesquioxane (Poss)Polymers," Current Opinion in Solid State and Materials Science, 8,2004, pp. 21 - 29.

(27) Wohl, C., Chen, L., Lin, Y. and Connell, J., "Phase-Segregated Copoly(Imide Butadiene)S for Friction Reduction," High Performance Polymers, manuscript in preparation,2011, pp.

(28) Wohl, C., "Synthesis, Characterization, Topographical Modification, and Surface Properties of Copoly(Imide Siloxane)S," High Performance Polymers, manuscript in preparation,2011, pp.

(29) Belcher, M., List, M., Ghose, S., Watson, K., Hopkins, J., Wohl, C. and Connell, J., "Effect of Laser Surface Preparation and Coupling Agent Composition on Durability of Adhesively Bonded Ti-6al-4v," 55th International SAMPE Symposium and Exhibition, Seattle, WA United States, 2010.

(30) Belcher, M., Wohl, C. and Connell, J., "Laser Surface Preparation and Bonding of Aerospace Structural Composites " 17th International Conference on Composite Materials, Edinburgh, United Kingdom, 2009.

(31) Mizes, H., Ott, M., Eklund, E. and Hays, D., "Small Particle Adhesion: Measurement and Control," Colloids and Surfaces A: Physicochemical and Engineering Aspects, 165,2000, pp. 11-23.

(32) Ebenstein, D., "Nano-Jkr Force Curve Method Overcomes Challenges of Surface Detection and Adhesion for Nanoindentation of a Compliant Polymer in Air and Water," Journal of Materials Research, 26,2011, pp. 1026-1035.

(33) Mullins, M., Micheals, L., Menon, V., Locke, B. and Ranade, M., "Effect of Geometry on Particle Adhesion," Aerosol Sci. Technol., 17,1992, pp. $105-118$.

(34) Wohl, C., Atkins, B. and Connell, J., "Method and Apparatus for the Quantification of Particulate Adhesion Forces on Various Substrates," National Aeronautics and Space Administration, TM-2011-217048, 2011.

(35) Zhao, Y., Shi, X. and Li, W., "Effect of Work of Adhesion on Nanoindentation," Reviews of Advanced Materials Science, 5,2003 , pp. 348 - 353.

(36) Israelachvili, J. Intermolecular and Surface Forces; Academic Press: Amsterdam, 2003.

(37) Kaelble, D. H., "Dispersion-Polar Surface Tension Properties of Organic Solids," Journal of Adhesion, 2,1970, pp. 66-81. 